\title{
Influência da Adição de Amido de Mandioca na Biodegradação da Blenda Polimérica PHBV/Ecoflex ${ }^{\circledR}$
}

\author{
Marilia Pellicano, Wagner Pachekoski, José A. M. Agnelli \\ Departamento de Engenharia de Materiais, UFSCar
}

Resumo: Os polímeros derivados do petróleo são amplamente utilizados devido à sua versatilidade, propriedades mecânicas e custo relativamente baixo, mas causam impacto ambiental como conseqüência da grande quantidade de resíduos lançados ao meio ambiente. Os problemas gerados pelo descarte de plásticos de origem petroquímica impulsionam o desenvolvimento, produção e aplicação de polímeros biodegradáveis. Entretanto, estes polímeros apresentam custo elevado e propriedades nem sempre satisfatórias. Portanto, neste trabalho fez-se um estudo do composto polimérico formado por poli(hidroxibutirato-co-valerato) - PHBV $\left(\right.$ Biocycle $\left.^{\circledR}\right)$, poli(butilenoadipato-tereftalato) - Ecoflex ${ }^{\circledR}$ e amido de mandioca. Este composto polimérico foi avaliado quanto ao índice de fluidez (MFI), Microscopia Eletrônica de Varredura (MEV), ensaios mecânicos de tração e de impacto, e ensaio de biodegradação. Os resultados obtidos nas diversas técnicas indicaram: que o amido de mandioca atua como uma carga inerte; que existe uma baixa adesão matriz/carga no composto polimérico e que a adição de amido aumenta a taxa de biodegradação do composto, em relação aos polímeros puros. No estudo realizado foi possível obter um material para aplicação no curto prazo, com um custo viável, propriedades físico-mecânicas adequadas e excelente taxa de biodegradação.

Palavras-chave: Polimeros biodegradáveis, biodegradação, PHBV, Biocycle ${ }^{\circledast}$, Ecoflex $^{\circledast}$, amido de mandioca.

\section{Influence of Cassava Starch Incorporation on the Biodegradability of the Polymeric Blend PHBV/Ecoflex ${ }^{\circledR}$}

\begin{abstract}
Plastic materials produced from petrochemicals are widely used due to their versatility, mechanical properties and low cost, but they cause environmental impact as a consequence of the accumulation of great amount of these conventional synthetic polymers. The problems caused by the waste disposal of these plastics have motivated the development, production and application of biodegradable polymers; however these polymers have high costs and non-suitable mechanical and physical properties. Therefore, a study of polymeric compounds made of poly(hydroxyburyrate-co-valerate) - PHBV, poly(butylyneadiapate-therefhtalate) - Ecoflex ${ }^{\circledR}$ and cassava starch has been done. This polymeric compound was evaluated using Melt Flow Index (MFI), Scanning Electron Microscopy (SEM), mechanical and biodegradation tests. The results indicate that the cassava starch acts as an inert filler, and that there is no significant adhesion between cassava starch and PHBV/Ecoflex ${ }^{\circledast}$. More importantly, adding cassava led to good biodegradation results. This study therefore demonstrates that it is possible to obtain viable materials for short-term applications, with adequate physical and mechanical properties and excellent biodegradation results.
\end{abstract}

Keywords: Biodegradable polymers, biodegradation, PHBV, Biocycle ${ }^{\circledast}$,Ecoflex ${ }^{\circledast}$, cassava starch.

\section{Introdução}

Nos últimos 50 anos os polímeros provenientes do petróleo têm sido muito utilizados e este sucesso se deve a sua versatilidade, propriedades mecânicas e custo relativamente baixo. Mas, o petróleo é uma matéria-prima exaurível e poluidora da natureza e desde a década de 80 houve uma grande conscientização em relação ao meio ambiente e uma ampla busca por materiais que apresentassem durabilidade durante o uso e degradabilidade após o descarte ${ }^{[1]}$. Com $\mathrm{o}$ intuito de minimizar problemas ambientais e atender às exigências do mercado, muito se têm investido numa nova classe de polímeros, os biodegradáveis, onde se destacam:
PLA - poli(ácido lático); PHB - poli(hidroxibutirato); PHBV - poli(hidroxibutirato-co-hidroxivalerato) e copoliésteres alifáticos aromáticos (AAC) biodegradáveis, tais como: Ecoflex ${ }^{\circledR}$ - poli(butilenoadiapato-tereftalato). Estes polímeros apresentam excelentes aplicações no setor de embalagens e filmes, devido a sua alta resistência à umidade, à gordura, à mudança de temperatura e propriedade de barreira a gases. Eles também são aplicáveis na medicina, no desenvolvimento de próteses, suturas, implantes e liberação controlada de drogas ${ }^{[2-4]}$. Por definição da ASTM, os polímeros biodegradáveis são aqueles que apresentam quebra de ligações químicas em suas cadeias poliméricas

Autor para correspondência: Marilia Pellicano, Departamento de Engenharia de Materiais, UFSCar, Caixa Postal 676, CEP: 13565-905, São Carlos, SP 
através de agentes biológicos, levando a uma fragmentação ou desintegração dos mesmos ${ }^{[2]}$.

No Brasil, são produzidas em escala semi-industrial cerca de 50 t/ano de PHBV a partir da fermentação do açúcar. Ele é um polímero de fonte renovável, completamente biodegradável e biocompatível (capaz de substituir alguns tecidos ou funções do corpo humano). Após o consumo, este PHBV pode ser descartado em aterros sanitários sem causar impacto ao meio ambiente, lá ele será biodegradado por bactérias e fungos, restando apenas água, gás carbônico e biomassa ${ }^{[5,6]}$.

O Ecoflex ${ }^{\circledR}$ é um polímero sintético biodegradável produzido pela empresa alemã Basf que em parceria com a Naturework lançou no mercado o Ecovio ${ }^{\circledR}$, blenda Ecoflex ${ }^{\circledR}$ PLA. Esta blenda pode ser utilizada na produção de embalagens, filmes, tubetes para reflorestamento, sacolas plásticas e embalagens para cosméticos ${ }^{[7]}$. Os poliésteres alifáticos aromáticos possuem capacidade instalada em torno de 30.000 t/ano com destaque para os polímeros Bionelle (Showa, japonesa), Eastar Bio (Eastman, americana) e Ecoflex ${ }^{\circledR}$ (Basf, alemã).

O mercado mundial de polímeros biodegradáveis cresceu de 18.400 t em 2006 para 24.350 t em 2007 e estima-se que venha a atingir algo em torno de $54.000 \mathrm{t}$ até 2012. O Brasil pode se tornar um grande produtor e exportador de polímeros biodegradáveis de fontes renováveis, pois dispõe de matéria-prima renovável e de baixo custo, além de possuir inúmeros grupos de pesquisa capazes de fornecer mão-deobra qualificada ${ }^{[7]}$.

Quando comparado ao polímero convencional, o alto custo na produção de polímeros biodegradáveis dificulta muito o seu emprego para a maioria das indústrias. O polietileno de baixa densidade amplamente utilizado em embalagens custa em média US\$2/kg, enquanto que um polímero biodegradável custa de US\$ 5 a $8 / \mathrm{kg}$. Com o intuito de baixar o custo e diminuir o impacto ambiental dos polímeros, adiciona-se em suas formulações um aditivo natural, abundante e degradável, como por exemplo o amido. No Brasil a Basf e a Corn Products lançaram o Ecobras ${ }^{\circledR}$, composto por Ecoflex ${ }^{\circledR} /$ amido $^{[5]}$.

Desta maneira, objetiva-se neste trabalho o estudo da incorporação de amido em uma mistura de PHBV e Ecoflex ${ }^{\circledR}$ visando a obtenção de um material para aplicação a curto prazo, a um custo viável, com propriedades físico-mecânicas adequadas e apresentando biodegradação, sendo a biodegradabilidade do mesmo em acompanhada a 60 e 120 dias por análise visual, perda de massa e propriedade mecânica.

\section{Materiais}

O PHBV utilizado corresponde ao lote FE-130, fornecido pela PHB Industrial S/A, com massa molar ponderal média de 113.000 g.mol ${ }^{-1}$.

O Ecoflex utilizado foi fornecido pela PHB Industrial. Tal produto é produzido pela Basf e possui massa molar ponderal média de 66.500 g. $\mathrm{mol}^{-1}$.

Para este trabalho optou-se pelo estudo de uma blenda polimérica de $70 \%$ de PHBV e 30\% de Ecoflex e a mudança de seu comportamento ao se incorporar $30 \%$ de amido de mandioca nesta blenda. Isto equivale a uma mistura com $49 \%$ de PHBV, $21 \%$ de Ecoflex e $30 \%$ de amido. A proporção de $30 \%$ de amido de mandioca foi adotada após um estudo realizado pelos autores, no qual se observou que este é o limite máximo de carga processável sem comprometimento das propriedades.

\section{Métodos}

\section{Preparação do composto polimérico}

$\mathrm{O}$ Ecoflex $^{\circledR} \mathrm{em}$ grânulos (produzido pela Basf), o PHBV em pó (lote FE-130, produzido e fornecido pela PHB Industrial S/A) e o amido de mandioca em pó (fornecido pela Cassava S/A) foram previamente secos em estufa com circulação de ar.

O amido utilizado, que possui umidade entre 12 e $14 \%$ e é altamente higroscópico, foi seco a $60{ }^{\circ} \mathrm{C}$ em estufa durante 24 horas antes dos processos de extrusão e injeção para evitar absorção de água e conseqüentemente interferência nas propriedades das misturas poliméricas.

O composto polimérico PHBV/Ecoflex ${ }^{\circledast} /$ amido de mandioca (49/21/30) foi processado em uma extrusora dupla rosca Imacom DRC 30:40 IF com diâmetro (D) de $30 \mathrm{~mm}$ e razão L/D de 40. Para a realização dos ensaios mecânicos o material foi submetido ao processo de injeção, utilizando a injetora Arburg All Rounder modelo 270V 300-120. A pressão e o tempo de recalque foram de 200 bar e 4 segundos, respectivamente. O tempo de resfriamento foi de 32 segundos e a temperatura nas zonas de aquecimento entre $145 \mathrm{e}$ $155^{\circ} \mathrm{C}$.

\section{Ensaio de índice de fluidez (MFI)}

A blenda PHBV/Ecoflex ${ }^{\circledR}$ e o composto PHBV/Ecoflex ${ }^{\circledR /}$ amido de mandioca foram caracterizados quanto ao índice de fluidez, utilizando-se um aparelho DSM modelo MI-1 nas condições: temperatura $=190^{\circ} \mathrm{C}$, carga $=2,16 \mathrm{~kg}$ e tempo de corte $=10$ segundos; especificadas pela norma ASTM D1238-04 $4^{[8]}$.

\section{Ensaios mecânicos}

A blenda PHBV/Ecoflex ${ }^{\circledR}$ e o composto polimérico PHBV/Ecoflex ${ }^{\circledR} /$ amido de mandioca foram caracterizados por ensaios de tração e impacto Izod, com entalhe. O ensaio de tração foi realizado em uma Máquina Universal de ensaios Instron, modelo 5569. Este ensaio está de acordo com a norma ASTM D638-04 ${ }^{[9]}$ na qual a distância entre garras $\left(\mathrm{l}_{0}\right)$ é igual a $115 \mathrm{~mm}$, a velocidade de ensaio é de $5 \mathrm{~mm} / \mathrm{min}$, a célula de carga é de $50 \mathrm{kN}$ e os corpos de prova apresentam as seguintes dimensões: comprimento igual a $165 \mathrm{~mm}$; espessura igual a $3,28 \mathrm{~mm}$; comprimento útil igual a $57,0 \mathrm{~mm}$ e largura da seção estreita igual a 13,0. O ensaio de impacto Izod foi realizado em uma Máquina de impacto Ceast code 6545/000 modelo RESIL com um pêndulo de 2J, seguindo 
a norma ASTM D256-05 ${ }^{[10]}$. Foram ensaiados 10 corpos de prova, sendo que a média aritmética das resistências da amostra ensaiada representa o valor da resistência ao impacto. Segundo a norma, os corpos de prova na forma de barras retangulares devem apresentar as seguintes dimensões: comprimento igual a $63,5 \mathrm{~mm}$; largura igual a $12,7 \mathrm{~mm}$; espessura igual a $3,28 \mathrm{~mm}$ e entalhe igual a $2,5 \mathrm{~mm}$.

\section{Microscopia eletrônica de varredura (MEV)}

Esta análise foi realizada no CCDM (Centro de Caracterização e Desenvolvimento de Materiais - UFSCar/UNESP) utilizando-se o Microscópio Eletrônico Leica, modelo Stereoscan 440.

\section{Ensaio de biodegradação}

O ensaio de biodegradação foi realizado no Laboratório de Polímeros Biodegradáveis do DEMa - UFSCar utilizandose as normas ASTM D6003 e ASTM G160 ${ }^{[11,12]}$. O solo utilizado é um composto orgânico humificado à base de esterco de aves e materiais orgânicos de origem vegetal, fornecido pela Provaso Indústria e Comércio de Fertilizantes Orgânicos S/S Ltda.

Para este ensaio de biodegradação foram preparados dois dispositivos de ensaio de biodegradação, para 60 e 120 dias, sendo que cada dispositivo continha 5 amostras (replicatas). Foram utilizados corpos de prova de impacto, seguindo as especificações da norma ASTM D256 ${ }^{[10]}$. A água perdida durante o ensaio, devido à evaporação, foi reposta semanalmente. Ao final de cada ensaio, as amostras degradadas foram lavadas cuidadosamente com água e secas em temperatura ambiente. As amostras biodegradadas foram avaliadas quanto ao aspecto visual, perda de massa e resistência ao impacto Izod, com entalhe.

\section{Resultados e Discussão}

A partir do ensaio de índice de fluidez foi possível observar que o amido de mandioca diminui o índice de fluidez do composto, atuando como o equivalente a uma carga tradicional, conforme Tabela 1. Segundo Rabello, M. ${ }^{[13]}$ as cargas inertes aumentam a viscosidade do material fundido,

Tabela 1. Resultado da análise de índice de fluidez (MFI).

\begin{tabular}{lc}
\hline \multicolumn{1}{c}{ Composições poliméricas } & $\begin{array}{c}\text { Índice de fluidez } \\
\text { (g/10 minutos) }\end{array}$ \\
\hline PHBV/Ecoflex $^{\circledR}(70 / 30)$ & 65,6 \\
PHBV/Ecoflex & /AMA (49/21/30) \\
\hline
\end{tabular}

dificultando o processamento, e normalmente diminuem a resistência ao impacto e a resistência à fadiga, contribuindo com uma maior propagação de trincas.

Analisando a Tabela 2 nota-se que a adição de amido torna o composto mais rígido (aumento do módulo de elasticidade) e menos resistente (diminuição da tensão de ruptura) que a blenda, ou seja, a adição de $30 \%$ de amido de mandioca à blenda PHBV/Ecoflex ${ }^{\circledR}$ (70/30) fragiliza o composto polimérico, conforme Figura 1. A adição de amido leva a uma redução das propriedades mecânicas devido à interface matriz/ carga com baixa compatibilidade e a não ocorrência do mecanismo de molhamento (as camadas de água existentes na superfície do amido não permitem a remoção do ar presente na interface $)^{[14-16]}$. Segundo Iovino, R. et al. ${ }^{[17]}$, o amido age como um concentrador de tensão no composto, induzindo trincas durante o ensaio de tração e diminuindo a resistência à tração resultante.

Analisando a micrografia obtida por MEV (Figura 2) é possível notar a formação de aglomerados de amido e vazios. Estes aglomerados são formados naturalmente pela carga impedindo que a matriz a envolva completamente, concentrando tensões e reduzindo as propriedades mecânicas ${ }^{[13]}$. Desta maneira, este comprovou a baixa adesão matriz/carga e a não ocorrência do mecanismo de molhamento no composto PHBV/Ecoflex ${ }^{\circledR} /$ amido mandioca $(49 / 21 / 30)$.

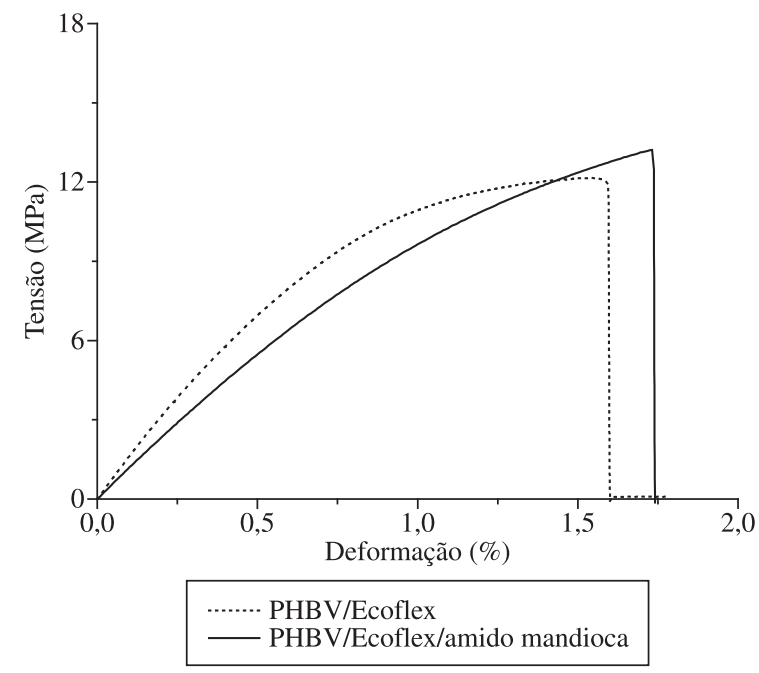

Figura 1. Curvas médias de tensão $\times$ deformação da blenda PHBV/Ecoflex ${ }^{\circledR}$ e do composto PHBV/ Ecoflex ${ }^{\circledast} /$ amido mandioca.

Tabela 2. Propriedades mecânicas em tração da blenda PHBV/Ecoflex ${ }^{\circledR}$ e do composto PHBV/ Ecoflex ${ }^{\circledast} /$ amido mandioca.

\begin{tabular}{lcc}
\hline \multicolumn{1}{c}{ Propriedades } & \multicolumn{2}{c}{ Composição polimérica } \\
\cline { 2 - 3 } & $\begin{array}{c}\text { PHBV/ Ecoflex } \\
\text { (70/30) }\end{array}$ & $\begin{array}{c}\text { PHBV/ Ecoflex }{ }^{\otimes / a m i d o ~} \\
\text { mandioca (49/21/30) }^{\circledR}\end{array}$ \\
\hline Módulo de elasticidade, em tração (GPa) & $1,14 \pm 0,01$ & $1,51 \pm 0,01$ \\
Tensão de escoamento, em tração (MPa) & $12,92 \pm 0,70$ & $12,10 \pm 0,12$ \\
Tensão na ruptura, em tração (MPa) & $13,21 \pm 0,48$ & $12,10 \pm 0,13$ \\
Deformação no ponto de escoamento (\%) & $1,7 \pm 0,1$ & $1,5 \pm 0,1$ \\
Deformação na ruptura, em tração (\%) & $1,8 \pm 0,1$ & $1,7 \pm 0,2$ \\
\hline
\end{tabular}




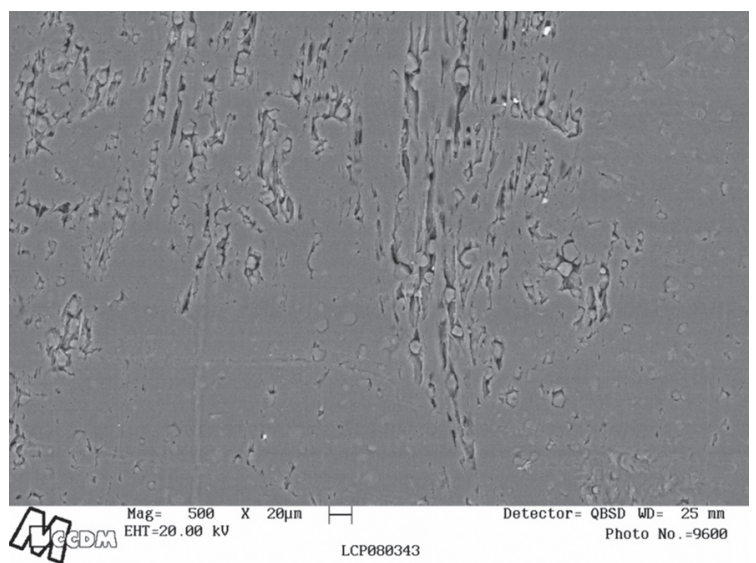

Figura 2. Micrografia do composto PHBV/Ecoflex ${ }^{\circledR} /$ amido mandioca (49/21/30), com aumento de 500x.

Por outro lado, os resultados do ensaio de resistência ao impacto Izod, com entalhe, mostram que o composto PHBV/ Ecoflex ${ }^{\circledast} /$ amido de mandioca $(49 / 21 / 30)$ apresentou-se tão tenaz quanto à blenda PHBV/Ecoflex ${ }^{\circledR}$ (70/30), indicando que a adição de amido não levou a uma diminuição na resistência ao impacto, conforme Tabela 3.

A análise visual dos corpos de provas a 120 dias após o ensaio de biodegradação mostra que o composto PHBV/ Ecoflex ${ }^{\circledR} /$ amido de mandioca (49/21/30) apresenta pontos ou regiões de pigmentação marrom-escuro (regiões circuladas da Figura 3 [a120]) e aspecto rugoso, enquanto que a blenda PHBV/Ecoflex ${ }^{\circledR}(70 / 30)$ apresenta pontos ou regiões de pigmentação vermelha (regiões circuladas da Figura 4 [a120]) e superfície lisa. A presença de regiões de pigmentação e a rugosidade presentes na superfície dos corpos de prova são decorrentes do processo de biodegradação. Estas regiões de pigmentação vermelha já haviam sido observados por Frank, H. K. ${ }^{[18]}$, no qual o fungo Serratia marcescens colonizou garrafas de leite feitas de polímeros sintéticos, tornando-as vermelhas, sendo que esta coloração não é removível, nem mesmo com lavagem intensa. A partir da análise das Figuras 3 e 4 é possível notar que o composto foi mais degradado que a blenda.

A Tabela 4 exibe os resultados de perda de massa para a blenda PHBV/Ecoflex ${ }^{\circledR}$ (70/30) e para o composto PHBV/ Ecoflex ${ }^{\circledast} /$ amido de mandioca (49/21/30). Segundo a análise da Tabela 3, nota-se que a adição de amido de mandioca aumenta a perda de massa, indicando um aumento na biodegradabilidade do composto em relação à blenda.

A Figura 5 exibe os resultados de resistência ao impacto Izod, com entalhe, para os corpos de prova antes (tempo zero) e depois (60 e 120 dias) do ensaio de biodegradação. O

Tabela 3. Resistência ao Impacto Izod, com entalhe, para a blenda PHBV/ Ecoflex ${ }^{\circledR}$ e composto PHBV/Ecoflex ${ }^{\circledR} /$ amido mandioca.

\begin{tabular}{lc}
\hline \multicolumn{1}{c}{ Composições poliméricas } & $\begin{array}{c}\text { Resistência ao impacto izod, } \\
\text { com entalhe }\left(\text { J.m }^{-1}\right)\end{array}$ \\
\hline PHBV/Ecoflex $^{\circledR}(70 / 30)$ & $24,2 \pm 0,9$ \\
PHB V/Ec oflex ${ }^{\circledR / a m i d o ~}$ & $24,5 \pm 0,4$ \\
mandioca $(49 / 21 / 30)$ & \\
\hline
\end{tabular}

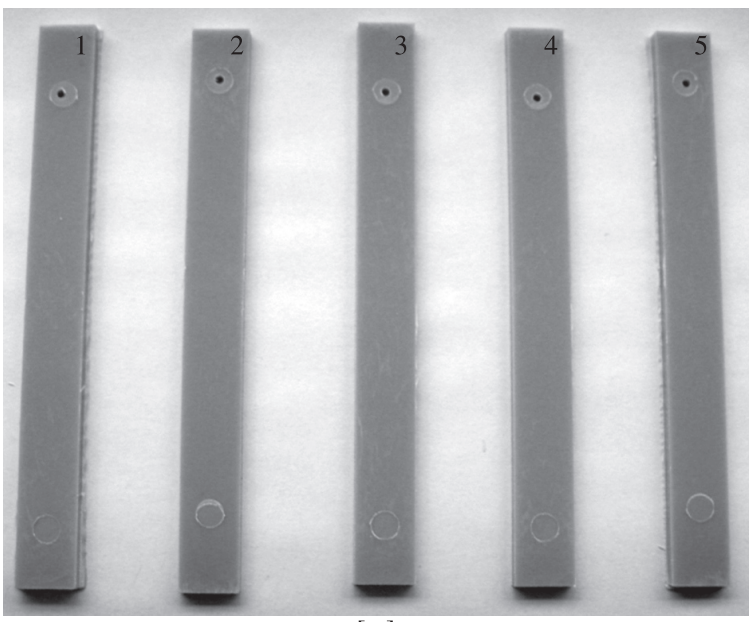

$\left[\mathrm{a}_{0}\right]$

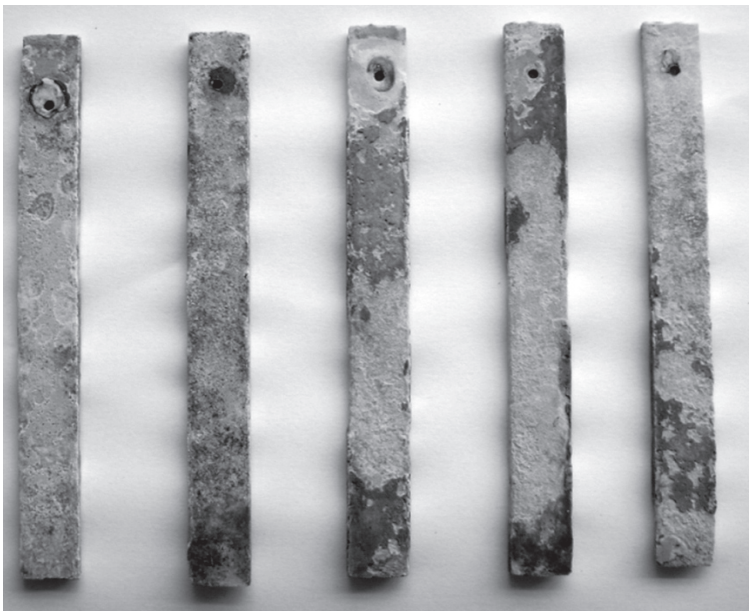

$\left[\mathrm{a}_{60}\right]$

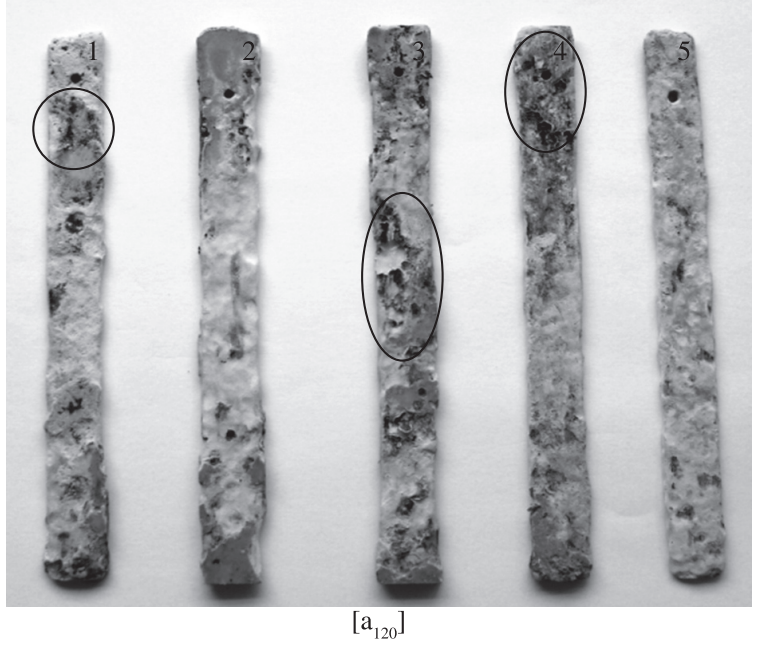

Figura 3. Fotos do composto PHBV/Ecoflex ${ }^{\circledast} /$ amido mandioca (49/21/30) no tempo zero $\left[\mathrm{a}_{0}\right]$, a $60\left[\mathrm{a}_{60}\right]$ e $120\left[\mathrm{a}_{120}\right]$ dias de ensaio de biodegradação.

composto polimérico apresentou melhor resultado de perda da tenacidade em relação à blenda, conforme Figura 5, indicando mais uma vez que a adição de amido de mandioca intensifica a biodegradação em solo. A diminuição da tenacidade durante o processo de biodegradação ocorre devido à 

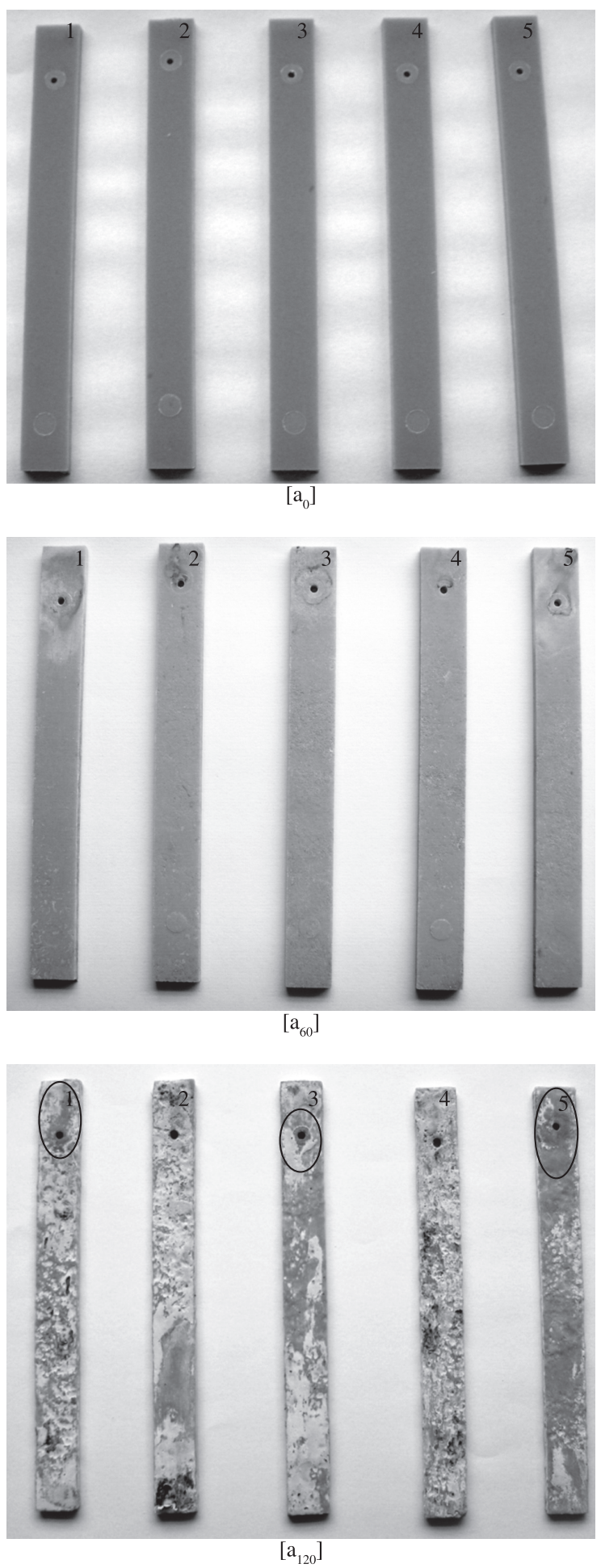

Figura 4. Fotos da blenda PHBV/Ecoflex ${ }^{\circledast}(70 / 30)$ no tempo zero $\left[a_{0}\right]$, a $60\left[\mathrm{a}_{60}\right]$ e $120\left[\mathrm{a}_{120}\right]$ dias de ensaio de biodegradação.

Tabela 4. Resultado do ensaio de Perda de massa para a blenda PHBV/ Ecoflex ${ }^{\circledR}$ e o composto PHBV/ Ecoflex ${ }^{\circledast} /$ amido mandioca.

\begin{tabular}{lcc}
\hline \multicolumn{1}{c}{$\begin{array}{c}\text { Composições } \\
\text { poliméricas }\end{array}$} & \multicolumn{2}{c}{ Perda de massa (\%) } \\
\cline { 2 - 3 } & 60 dias & $\mathbf{1 2 0}$ dias \\
\hline PHBV/Ecoflex $^{\circledR}(70 / 30)$ & $5,2 \pm 0,1$ & $24,1 \pm 0,1$ \\
PHBV/Ecoflex ${ }^{\circledR} /$ amido & $5,8 \pm 0,2$ & $44,0 \pm 0,3$ \\
mandioca $(49 / 21 / 30)$ & & \\
\hline
\end{tabular}

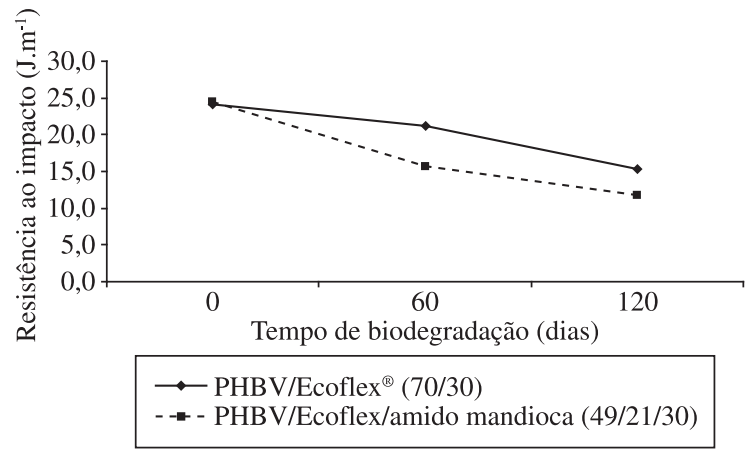

Figura 5. Tempo de biodegradação $\times$ Resistência ao impacto Izod, com entalhe, para a blenda PHBV/Ecoflex ${ }^{\circledR}$ e composto PHBV/Ecoflex ${ }^{\circledR} /$ amido mandioca.

presença de vazios tanto na superfície quanto no interior dos corpos de prova, facilitando a propagação de trincas e diminuindo as propriedades mecânicas ${ }^{[19]}$.

\section{Conclusões}

A análise visual, a perda de massa e a resistência ao impacto Izod, com entalhe, mostraram-se técnicas simples e muito úteis para avaliar a biodegradação de blendas e compostos poliméricos biodegradáveis.

Com base nos resultados obtidos, pode-se concluir que o amido de mandioca atua como uma carga inerte, e que a adição de $30 \%$ do mesmo à blenda $\mathrm{PHBV} /$ Ecoflex $^{\circledR}$ fragiliza o composto PHBV/Ecoflex ${ }^{\circledast} /$ amido de mandioca (49/21/30) devido a dois fatores: a baixa compatibilidade da interface matriz/carga e a não ocorrência do mecanismo de molhamento da carga pela matriz, uma vez que as camadas de água existentes na superfície do amido não permitem a remoção do ar presente na interface. Mas, em contra partida, a presença do amido aumenta a velocidade de biodegradação do composto.

\section{Agradecimentos}

Os autores agradecem à PHB Industrial S/A, ao Laboratório de Polímeros Biodegradáveis do DEMa-UFSCar e ao CNPq.

\section{Referências Bibliográficas}

1. Callister, W. D. - "Materials Science and Engineering: an Introduction", John- Wiley, New York (1994).

2. Mei, L. H. I. - "Polímeros biodegradáveis" in: Biomateriais: Fundamentos e Aplicações, Oréfice, R. L.; Pereira, M. M.; Mansur, H. S. (Eds.), Cultura Médica, Rio de Janeiro (2006).

3. Rosa, D. S. \& Pantano Filho, R. - "Biodegradação: um Ensaio com Polímeros", Moara Editora, Itatiba (2003). 
4. Baumann, M. G. - "Plastics Solid Waste Management: the Role of Source Reduction, Re-Use, Biodegradability and Incineration Future”, in: Proceedings of the Seminars at the Society of Plastics Engineer, p.23, Atlanta - USA, (1998).

5. Chandra, R. \& Rutsgi, R. - Polymeros Science, 23, p.1273 (1998).

6. Morais, J. A. - Reciclagem Moderna, 8, p.24 (2007).

7. Raupp, A. - Embalagem Marca, p.57 (2007).

8. ASTM D1238-04 - "Standard test method for melt flow rates of thermoplastics by plastometer". Philadelphia, 2001.

9. ASTM D638-03 - "Standard test method for tensile properties of plastics". Philadelphia, 2001.

10. ASTM D256-05 - "Standard test methods for determining the izod pendulum impact for plastics". Philadelphia, 2001.

11. ASTM D6003-96 - "Standard method for determining weight loss from plastic materials exposed to simulated municipal solid-wast (MSM) aerobic compost environment”. Philadelphia, 1996.
12. ASTM G160-98 - "Standart practice for evaluating microbial susceptibility of nonmetallic materials by laboratory soil burial". Philadelphia, 1998.

13. Rabello, M. - "Aditivação de polímeros", Artliber, São Paulo (2000).

14. Koller, I. \& Owen, A. J. - Polym. Intern., 39, p.175 (1996).

15. Caraschi, J. C. \& Leao, A. J. "Composites of polyhydroxybutyrate with Natural Fibers: Preparation and Caracterization”, in: Anais do $6^{\circ}$ Congresso Brasileiro de Polímeros, p. 4, Gramado - RS, (2001).

16. Koenig, M. F. \& Huang, S. J. - Polym., 36, p.1877 (1995).

17. Iovino, R.; Zullo, R.; Rao, M. A.; Cassar, L. \& Gianfreda, L. - Polym. Degrad. Stabil., 93, p.147 (2008).

18. Frank, H. K. - "Microbial Pigmentation", in: Anais do $8^{\circ}$ Forum de Microbiologia, p.339, Leipizig - DE, (1984)

19. Luo, S. \& Netravali, A. N. - Polym. Degrad. Stab., 80, p.59 (2003).
Enviado: 07/07/08

Reenviado: 22/04/09

Aceito: 04/05/09 